\title{
A Network Pharmacology Approach to Uncover the Molecular Mechanisms of Herbal Formula Kang-Bai-Ling for Treatment of Vitiligo
}

\author{
Manyuan Xu, ${ }^{1}$ Jianxin Shi, ${ }^{2}$ Zhongsheng Min, ${ }^{2}$ Hongliu $\mathrm{Zhu}^{3}$ and Weiguo Sun ${ }^{1}{ }^{1}$ \\ ${ }^{1}$ Department of Dermatology, The Affiliated Huaian No. 1 People's Hospital of Nanjing Medical University, \\ Huaian 223300, China \\ ${ }^{2}$ Department of Dermatology, Jiangsu Province Hospital of Chinese Medicine, Nanjing 210029, China \\ ${ }^{3}$ Department of Dermatology, Jiangyin Hospital of Traditional Chinese Medicine, Jiangyin 214400, China \\ Correspondence should be addressed to Weiguo Sun; sls1242@163.com
}

Received 8 July 2019; Revised 15 September 2019; Accepted 4 October 2019; Published 3 November 2019

Academic Editor: Darren R. Williams

Copyright (C) 2019 Manyuan Xu et al. This is an open access article distributed under the Creative Commons Attribution License, which permits unrestricted use, distribution, and reproduction in any medium, provided the original work is properly cited.

Background. Kang-bai-ling (KBL), a Chinese patent medicine, has been demonstrated as an effective therapy for vitiligo in China. However, the pharmacological mechanisms of KBL have not been completely elucidated. Methods. In this study, the potential multicomponent, multitarget, and multipathway mechanism of KBL against vitiligo was clarified by using network pharmacology-based strategy. In brief, potential targets of KBL were collected based on TCMSP databases, followed by network establishment concerning the interactions of potential targets of KBL with well-known therapeutic targets of vitiligo by using protein-protein interaction (PPI) data. As a result, key nodes with higher level of seven topological parameters, including "degree centrality (DC)," "betweenness centrality (BC)," "closeness centrality (CC)," "eigenvector centrality (EC)," "network centrality (NC)," and "local average connectivity (LAC)" were identified as the main targets in the network, followed by subsequent incorporation into the ClueGO for GO and KEGG signaling pathway enrichment analysis. Results. In accordance with the topological importance, a total of 23 potential targets of $\mathrm{KBL}$ on vitiligo were identified as main hubs. Additionally, enrichment analysis suggested that targets of KBL on vitiligo were mainly clustered into multiple biological processes (associated with DNA translation, lymphocyte differentiation and activation, steroid biosynthesis, autoimmune and systemic inflammatory reaction, neuron apoptosis, and vitamin deficiency) and related pathways (TNF, JAK-STAT, ILs, TLRs, prolactin, and NF- $\kappa$ B), indicating the underlying mechanisms of KBL on vitiligo. Conclusion. In this work, we successfully illuminated the "multicompounds, multitargets" therapeutic action of KBL on vitiligo by using network pharmacology. Moreover, our present outcomes might shed light on the further clinical application of KBL on vitiligo treatment.

\section{Background}

Vitiligo, a progressive and acquired depigmentation disease, is characterized by the presence of circumscribed white macules on the skin [1]. An estimated $1-4 \%$ of the global populations are influenced by vitiligo [2], with certain subgroups at higher risk (up to 2-3\%) [3]. In terms of the mechanism of pigmentation loss, a series of speculations have been proposed, including oxidative stress, autoimmunity, injured melanocyte migration, and/or proliferation as well as genetic and neural theories [4-7].
The present clinical therapeutic strategies mainly focus on the enhanced melanogenesis of melanocytes in skin lesions and the restoration of skin color to the normal level [8]. In addition, vitamin $\mathrm{D}$ analogs, excimer laser, and steroid therapy aim at the restoration of pigmentation [9-11], the successful rates of which, however, are restricted by the failure to follow the primary pathogenesis of vitiligo. Of note, the administration of psoralens combined with subsequent exposure to ultra-violet light (sun light) is considered as one of the most common therapies of vitiligo, nevertheless only $61 \%$ of patients could obtain over $25 \%$ 
repigmentation [12]. Moreover, the above therapy could induce serious adverse events, including phototoxic response, perioral dermatitis, cutaneous atrophy, and longterm carcinogenic risk [13]. To this end, accumulative efforts have been made to identify novel therapeutic medicine and to evaluate multitargets, in order to examine the complicated network of the therapeutic mechanism of vitiligo.

Traditional Chinese medicine (TCM), a comprehensive medicinal system, plays a key role in maintaining health for Asian population [14]. Accumulative prevalence has been gained on TCM in western countries due to the robust therapeutic effects and relatively few side effects. According to the theory of traditional Chinese herbal medical science, TCM is considered as promising preventive and therapeutic sources for complicated disorders, including vitiligo [15-18].

Kang-bai-ling (KBL), a Chinese patent medicine, consists of five medicinal herbs, including Psoraleae Fructus (Buguzhi, BGZ), Angelicae Dahuricae Radix (Baizhi, BZ), Stephaniae Tetrandrae Radix (Fangji, FJ), Mume Fructus (Wumei, WM), and Glycyrrhizae Radix et Rhizoma (Gancao, GC). KBL, first proposed by Professor Min Zhongsheng in 1990s, has been utilized to treat a great number of patients with vitiligo ever since. Previous studies have indicated the good curative effect of KBL in clinics [19]. The mechanism of KBL against vitiligo is related to the up-regulated expression of tyrosinase in melanin cells, enhanced activity and induced synthesis of melanin [20]. However, the scientific basis as well as potential pharmacological mechanisms of KBL is still unclear and needs further investigations.

The therapeutic effects of the majority of TCM drugs are generally regulated by diverse targets and pathways in human body due to the complicated active components of TCM, which could not easily be accurately determined through traditional approaches. Thus, it is urgent to develop novel and appropriate strategies [21]. Herein, in this study, a comprehensive approach (a combination of multiple network-based computational and algorithm-based approaches) was utilized, by combining prediction of active compounds based on multiple pharmacokinetic parameters and excavation of diverse drug targets and network analysis, aiming at illumination of the underlying mechanisms of $\mathrm{KBL}$ on vitiligo.

\section{Methods}

2.1. Data Preparation. Data for all ingredients of each herb in KBL were collected from the Chinese Academy of Sciences Chemistry Database [22] (http://www.organchem. csdb.cn/scdb/default.asp), Traditional Chinese Medicine System Pharmacology Database [23] (http://lsp.nwu.edu.cn/ tcmsp.php), and relevant literatures, followed by subsequent addition into the ingredient database.

2.2. $O B$ and Drug-Likeness Screening. OB prescreening, defined as the distribution degree of an oral dose of drug into bloodstream, is one of the most requisite premises in terms of oral drug discovery as well as clinical application [24]. Additionally, drug-likeness, which is defined as a qualitative concept for assessment of the structural similarity of compounds with clinical therapeutics in the DrugBank database, is determined early after drug discovery [25]. The detailed calculations of these two parameters have been reported by Wang et al. Briefly, $\mathrm{OB}=30 \%$ along with druglikeness index $=0.18$ was defined as the cutoff value for the selection of candidate compounds [26]. In our study, several compounds, including psoralen and angelicin, were initially omitted in line with these screening criteria. Nevertheless, they were still included into candidate compounds for further analysis, due to their widely acknowledged pharmacological activities [27].

2.3. Potential Drug Targets Prediction for KBL. The identification of targets is considered as a pivotal stage during drug exploitation. Herein, systematic drug-targeting approach, which was proposed by Li et al. [28], was utilized for accurate determination of potential targets for medical compositions.

2.4. Collection of Known Vitiligo-Related Targets. Known vitiligo-associated targets were acquired from five existing resources: (1) MalaCards human disease database [29] (https://www.malacards.org); (2) OMIM database [30] (https://omim.org); (3) Therapeutic Target Database [31] (http://bidd.nus.edu.sg/group/cjttd/); (4) DrugBank database [32] (https://www.drugbank.ca); (5) Genetic Association Database [33] (https://geneticassociationdb.nih.gov). The detailed information of these known therapeutic targets is summarized in Table S3. Finally, 98 known vitiligo-related targets were selected after redundancy deletion.

2.5. PPI Data. Afterwards, PPI data were retrieved from a prevalently used Cytoscape plugin BisoGenet [34] through six present PPI datasets, including Biological General Repository for Interaction Datasets (BioGRID), Database of Interacting Proteins (DIP), Biomolecular Interaction Network Database (BIND), Molecular INTeraction Database (MID), Human Protein Reference Database (HPRD), and InAct. In addition, the detailed information of these PPI datasets is summarized in Table S6.

2.6. Network Construction. Based on the interaction data obtained from the Cytoscape plugin BisoGenet, we first constructed an interaction network of the known vitiligoassociated targets and potential drug targets of KBL, followed by further visualization using Cytoscape (Version 3.6.0).

2.7. Defining of Network Topological Feature Set. We then assessed the topological property of every node in the interaction network by calculating six parameters with a Cytoscape plugin CytoNCA [35]: "degree centrality (DC)," "betweenness centrality (BC)," "closeness centrality (CC)," "eigenvector centrality (EC)," "network centrality (NC)," and "local average connectivity (LAC)." The definitions and computational formulas of these parameters have been 
previously defined and represent the topological importance of a node in the network. These six parameters were representatives of the topological significance of each individual node of the entire network. To be specific, a larger quantitative value indicated the greater significance of the node in this network.

2.8. Gene Ontology and Pathway Enrichment Analysis. GO analysis: targets were performed in Omicshare [36] for more intensive understanding of their role in three categories: biological processes (BP), molecular function (MF), and cell component (CC). A $P$ value of $\leq 0.05$ indicated statistical significance, followed by applied hypergeometric examination to identify enriched GO terms.

ClueGO [37], a widely used Cytoscape plugin to visualize the nonredundant biological terms for large clusters of genes in a functionally grouped network, was used to reflect the enrichment analysis of 23 candidate targets of KBL. The present findings were categorized into two large clusters in this study: biological processes and the signaling pathway. ClueGO network, established by kappa statistics, was used to reflect the relationships of terms in accordance with the similarity of their related genes.

\section{Results}

3.1. Candidate Compound Screening for KBL. Accumulative efforts have been made to clarify the therapeutic mechanisms of TCM, however, with sluggish progress on the molecular level. Due to the unavailable effective methods specifically developed for identification of the active compounds in medicinal herbs, $\mathrm{OB}$ screening combined with drug-likeness assessment may be a feasible strategy. In our study, a total of 123 possible compounds with proper values of these two parameters were collected from the herbal constituents of KBL. In addition, 45 compounds (such as psoralen and angelicin) with lower values of $\mathrm{OB}$ or drug-likeness parameter, exhibiting massive pharmacological activities, were typical components of herbal drugs (reported in literature) and were also collected as the candidate active compounds. Thus, these compounds were chosen as candidate-active compounds as well. Finally, 168 compounds of five herbs were recognized "candidate compounds" (Table S1). To be specific, the numbers of candidate compounds in BGZ, BZ, FJ, WM, and GC was $13,38,9,13$, and 117, respectively. Among all the candidate compounds, 17 of them were distributed in more than one herb of $\mathrm{KBL}$, with validated diverse biological activities. For instance, linoleic acid, existing in almost all the three herbs except for FJ and GC, exerts potent antioxidant, anti-inflammatory, metabolic regulation, anticancer, and immune-regulatory activities [38]. Similarly, quercetin, ursolic acid, stigmasterol, and angelicin, which were distributed in at least two herbs of KBL, also exert various pharmacological properties, participating in the regulation of multiple physiological and pathological processes, such as inflammation, immune response, and stress process [39-42].
3.2. Potential Target Prediction for KBL. TCM formulas were generally effective in terms of preventive and curative effects on complicated disorders, entirely dependent on the combined effects of various compounds and targets. Thus, apart from the clarification of probable active compounds of KBL, we should explore the therapeutic targets as well. Herein, potential targets of candidate compounds were predicted based on the integration of chemical, genomic, and pharmacological data. For 168 candidate compounds, 299 potential targets were predicted (Table S2). The numbers of potential targets linked by BGZ, BZ, FJ, WM, and GC were $86,96,71,232$, and 275 , respectively. Despite the different numbers of targets related to each herb, significant overlaps were observed in the five herbs, indicating that diverse components in KBL may exert congeneric effects possibly by modulating the similar targets. For instance, both BGZ and BZ have been validated to affect the pathogenesis and progression of vitiligo through regulating immune- and inflammation-related pathways and promoting both adhesion and migration of melanocytes [43].

For further understanding of the roles of the potential targets involved in diverse biological processes, cellular component as well as molecular functions, preliminary GO (Gene Ontology) analysis was conducted with Omicshare, a free database based on DAVID, revealing that the potential targets were enriched in response to various types of stimuli (ROS, chemical, drugs, inflammatory factors and hormone), immune systems, cellular metabolism, cell-cell communication, cell growth, and death and development, all of which participated in the pathologic process of vitiligo (Figure 1 and Table S7) [44-47].

For more comprehensive understanding of $\mathrm{KBL}$ component-targets network from a systematic and holistic perspective, Cytoscape was used to construct a network map, which altogether consisted of 490 nodes as well as 4303 edges (Figure 2). To be specific, the degree of node indicated the number of edges or targets correlating with node based on the topological analysis. In our network, 93 candidate components were identified with a median $\geq 20$ degrees. To be specific, quercetin, kaempferol, and ursolic acid acted on 162, 72, and 61 targets, respectively, which were therefore considered as the critical bioactive ingredients in KBL. Quercetin has been demonstrated to decrease hydrogen peroxide-induced intracellular redox imbalance in melanocyte and thereby achieving repigmentation by regulating certain crucial pathways $[48,49]$. In addition, kaempferol and ursolic acid have also been validated to increase melanin content via positively regulating the activity of tyrosinase directly [50]. These findings might shed novel light on the pleiotropic effects of TCM herbs.

3.3. Collection of Known Vitiligo-Related Targets. Vitiligo is a polygenic predisposing disorder. Genetic-environmental interaction has been revealed to play a role in the pathogenesis of vitiligo. Herein, 98 vitiligo-related targets were retrieved from five present resources. Notably, 15 of the 


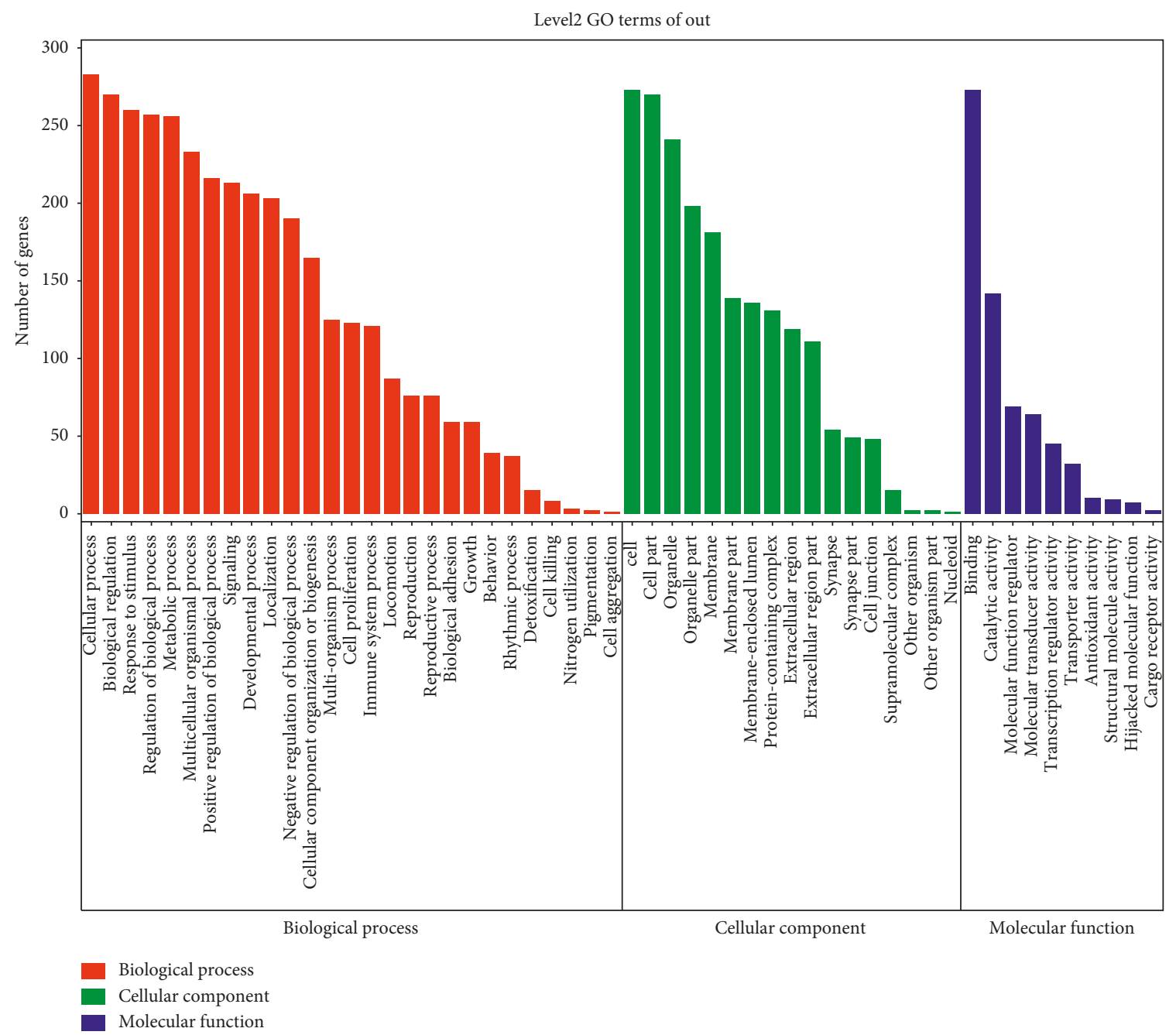

FIGURE 1: Gene ontology analysis of the potential targets of KBL. Potential targets of KBL was performed in Omicshare to gain more insights into their involvement in various Biological Processes (red section), Molecular Function (blue section) and Cell Component (green section). We considered a $P$ value cutoff of $\leq 0.05$ as significant and applied hypergeometric test to identify enriched GO terms. Terms of same category are ordered by $P$ values, left terms are more significant. Information of the number of involved genes in a term are shown in $y$-axis.

identified potential targets of the KBL were also the wellrecognized vitiligo disease (or therapeutic drugs)-related targets, indicating the potential therapeutics of this formula (Figure 3(a) and Table S4).

To understand the possible pharmacological mechanisms of KBL on vitiligo, GO and KEGG enrichment analyses were performed on the above-described 15 targets (Figures 3(b) and 3(c) and Tables S8 and S9). Consistent with the current pathological mechanism of vitiligo, they were mainly enriched in homeostatic process, cell growth and death, cellular metabolism, immune and inflammatory responses, and neuroendocrine signal conduction. Moreover, KBL was found to modulate vitiligo via the regulation of activity of multiple series enzymes such as tyrosinase (TYR), oxidoreductase (GSTP1, CAT, MPO, and GSTM1), and controlling signal transducer (NFE2L2). Furthermore, inflammatory factors and interleukin (IL) signaling pathway (IL4 and IL10), adaptive immune response (IFNG), and cell apoptosis (FASLG and TNF) also played critical roles in the clarification of the mechanisms of KBL on vitiligo. Notably, vitiligo has been accumulatively reported to suffer from uncontrolled neurocrine regulation, all of which affect every situation and bring greater challenges to vitiligo treatment [51]. In the present study, our analysis suggested that KBL might be of therapeutic effects on vitiligo by affecting catecholamine metabolism, neurotransmitter degradation (MAOA and $\mathrm{MAOB}$ ), and intracellular steroid hormone receptor signaling pathway (ESR1). Consistent with previous outcomes, we confirmed that KBL exerted a curative role on not only melanocytes but also neuroendocrine system from the network pharmacologic level. Vitiligo-related drugs causing systemic alterations would not only control vitiligo but might play also a therapeutic or side role on other disorders. By contrast, drugs regulating systemic alterations could affect vitiligo as well. Taken together, we hypothesized that vitiligo might respond towards systemic neuro-immunoinflammatory disorder in the skin tissue, which could be systematically controlled by KBL. 


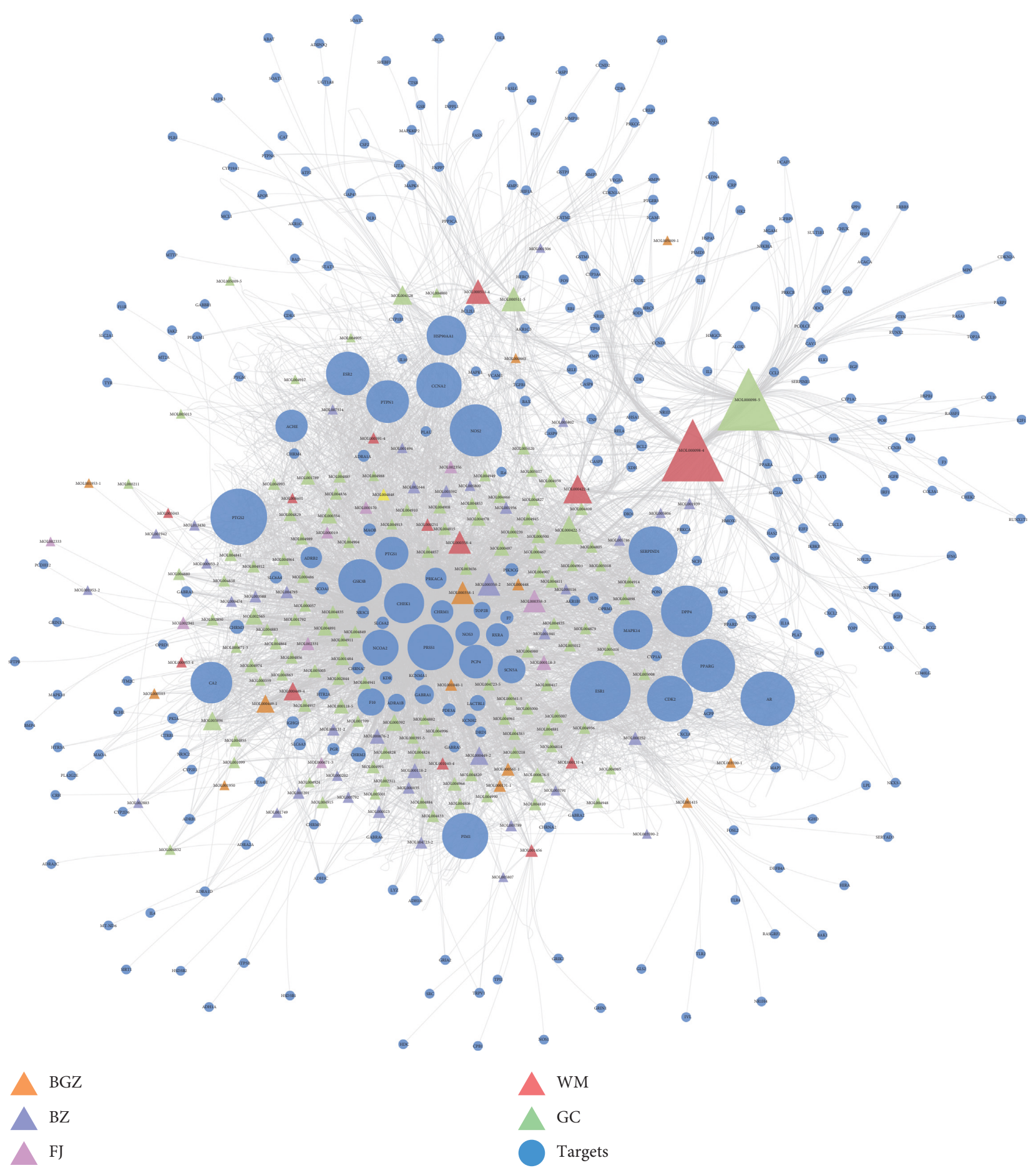

FIGURE 2: Construction of the KBL compound-potential target network. The compound-potential target network was constructed by linking the candidate compounds and their potential targets of the 5 herbs, which are constituents of KBL. The nodes representing candidate compounds are shown as polychrome triangle and the targets are indicated by blue circular.

3.4. Identification of Candidate Targets for KBL against Vitiligo. As indicated by recent evidences in network biology, disease-related genes or proteins do not work alone; instead, there are diverse interactive pathways and molecular networks on various levels. To clarify the pharmacological mechanism by which KBL ameliorates vitiligo, we constructed protein-protein networks that may reflect the behavior and properties of biological molecules. To this end, a potential target network (394 nodes and 24238 edges) and a defined vitiligo-associated target network (295 nodes and 14043 edges) were established by utilizing PPI data of these genes, respectively. We then merged these two networks to obtain a core protein-protein interaction (CPPI) network that consisted of 87 nodes and 1946 edges. Subsequently, candidate antivitiligo targets of KBL were screened by using the topological features of CPPI. A node with the topological 


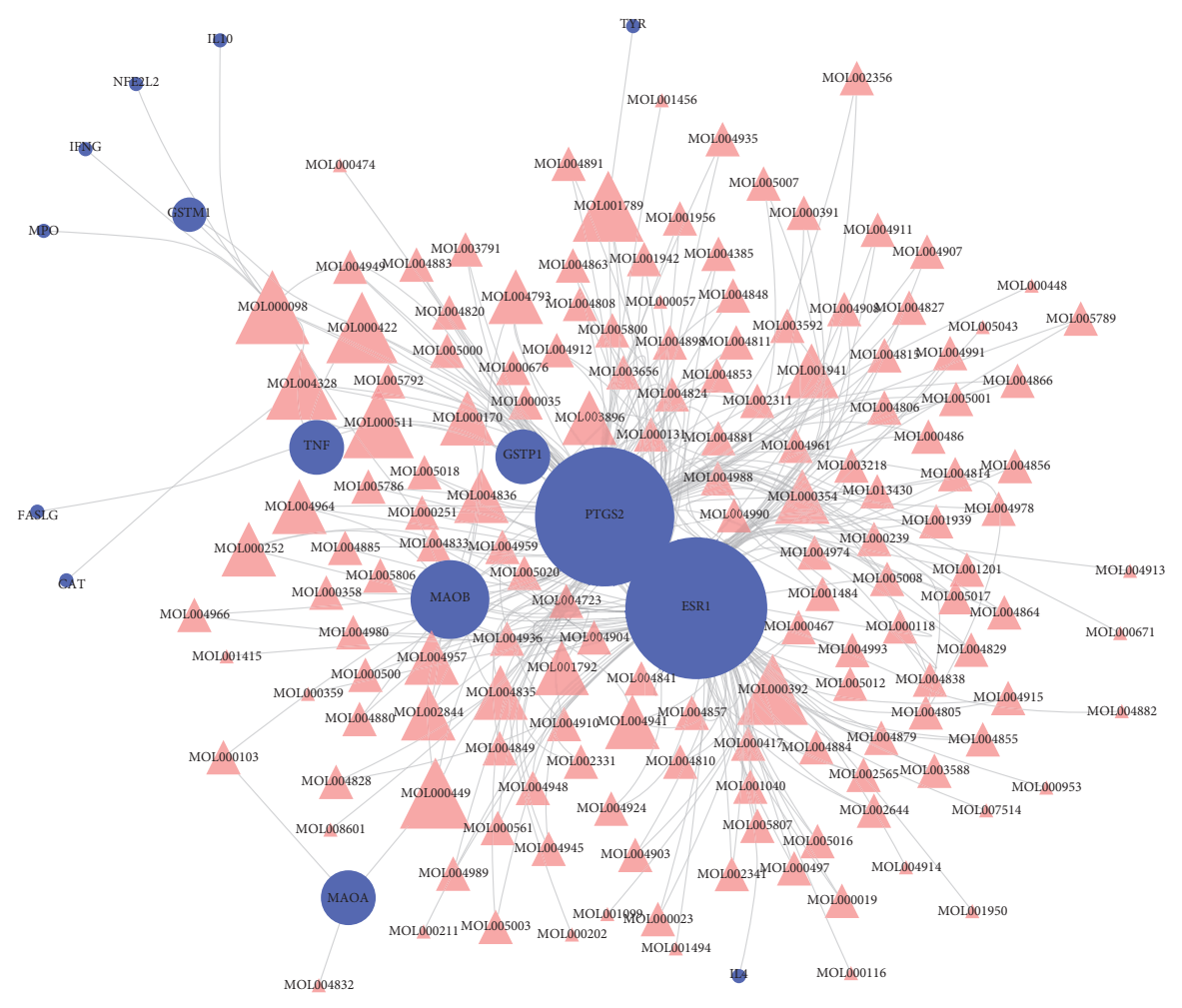

Targets

(a)

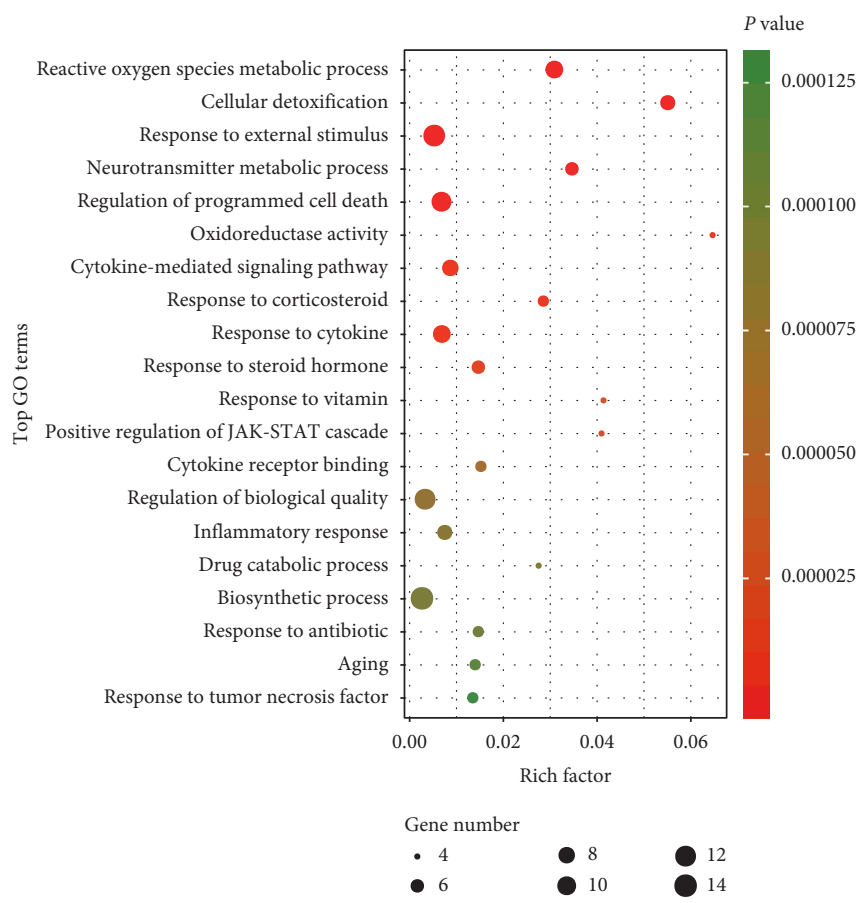

(b)

FIGURE 3: Continued. 


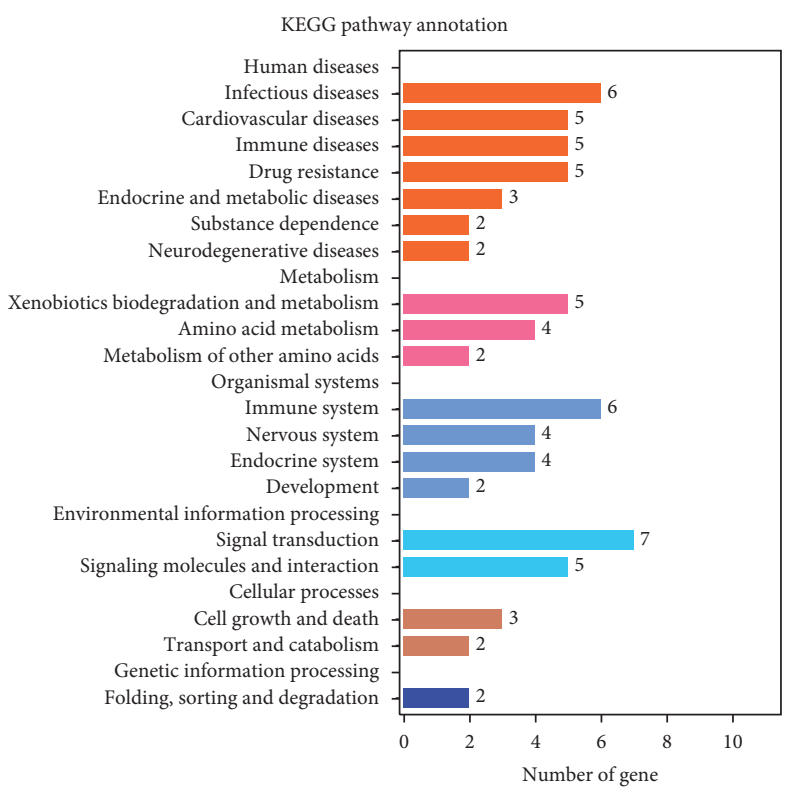

(c)

FIGURE 3: KBL shared 15 potential targets with known pathological course related targets of vitiligo. (a) The compound potential target network was constructed by linking the overlapped targets (between KBL potential and known vitiligo-related) and the homologous candidate compounds of KBL. The nodes representing candidate compounds are shown as pink triangle and the targets are presented as purple circular. (b) 15 overlapped targets (between KBL potential and known vitiligo-related) was performed in Omicshare to gain more insights into their involvement in various GO terms. We considered a $P$ value cutoff of $\leq 0.05$ as significant and applied hypergeometric test to identify enriched GO terms. Following chart shows an overview of the gene ontology analysis with up to 20 significantly enriched terms. (c) 15 overlapped targets (between KBL potential and known vitiligo-related) was performed in Omicshare to gain more insights into their involvement in KEGG pathways.

features exceeding that of median of all nodes was considered as a hub in the network (candidate targets in our study). As a result, the candidate targets were identified according to a widely used plugin CytoNCA, six topological features, including "DC," "BC," "CC," "EC," "NC," and "LAC." The median values of "DC," "BC," “CC," "EC," "NC," and "LAC" were 43, 23.3953542332676, 0.666666666666666, 0.103930160403251, 37.2721127301037 , and 35.15, respectively. Therefore, 23 candidate targets, with "DC" $>43$, "BC" > 23.3953542332676, "CC" $>0.666666666666666$, "EC" >0.103930160403251, "NC" > 37.27 21127301037, and "LAC" > 35.15, were identified. The detailed topological characteristics and the PPI network are shown in Table S5.

3.5. Enrichment Analysis of Candidate Targets for KBL against Vitiligo. To further reveal the probable role of 23 candidate targets, ClueGO, an extensively utilized Cytoscape plugin, was employed to find out interrelations of functional groups with their potential scientific connotation in biological networks, followed by division into GO biological processes as well as signaling pathway (Figure 4). To be specific, biological processes were mainly associated with DNA translation, lymphocyte differentiation and activation, STAT signal transduction, steriod biosynthesis, and peripheral nervous system myelin maintenance (Figure 4(a)). And multiple pathological processes and diseasees, including inflammatory cytokine stimulation, intracellular ROS accumulation, neuron apoptotic process, and vitamin deficiency, could indirectly affect the progression of vitiligo, providing potent evidence for our speculation that $\mathrm{KBL}$ exerted a curative role on vitiligo via systemic neuro-immunoinflammatory modulation from a genetic perspective.

The involved pathway was mainly composed of TNF, JAK-STAT, ILs, TLRs, NF- $\kappa$ B, prolactin, lymphocyte differentiation-related, and autoimmune and systemic inflammatory reaction-related pathways (Figure 4(b)). The above pathways could further be categorized into three representative modules, namely, immunoregulation, melanogenesis, and neuroendocrine regulation. Inflammation and immune mechanisms are prone to play a role in the melanocyte destruction of vitiligo, while ILs, TLRs, and JAKSTAT signaling pathways participate in regulating proinflammatory and immune reactions, as well as lymphocyte differentiation. In addition, destructive melanocytes are well-known characteristics of vitiligo. NF- $\kappa \mathrm{B}$, which is capable of decreasing apoptosis of vitiliginous keratinocytes, thereby makes contributions to the proliferation as well as differentiation of melanocytes, further promoting melanin biosynthesis. Moreover, there is intimate interaction between melanocytes and nerve fibers as well as extracellular matrix, while mediators might directly modulate the functions of melanocytes. Hyperactive prolactin pathway is likely to cause hyperproduction of $\mathrm{H}_{2} \mathrm{O}_{2}$, which is capable of not only changing calcium homeostasis to influencing vitiligo progression via the modulation of synaptic transmission, 


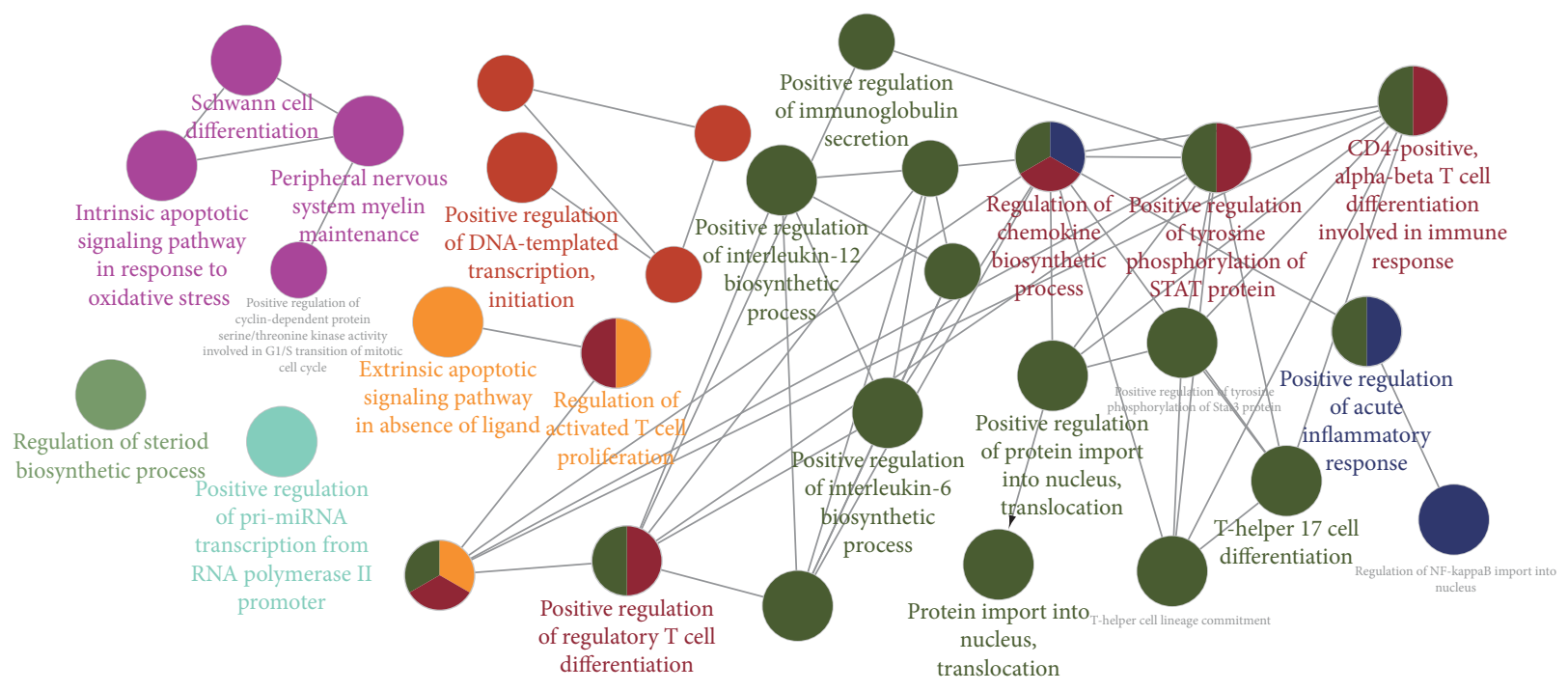

(a)

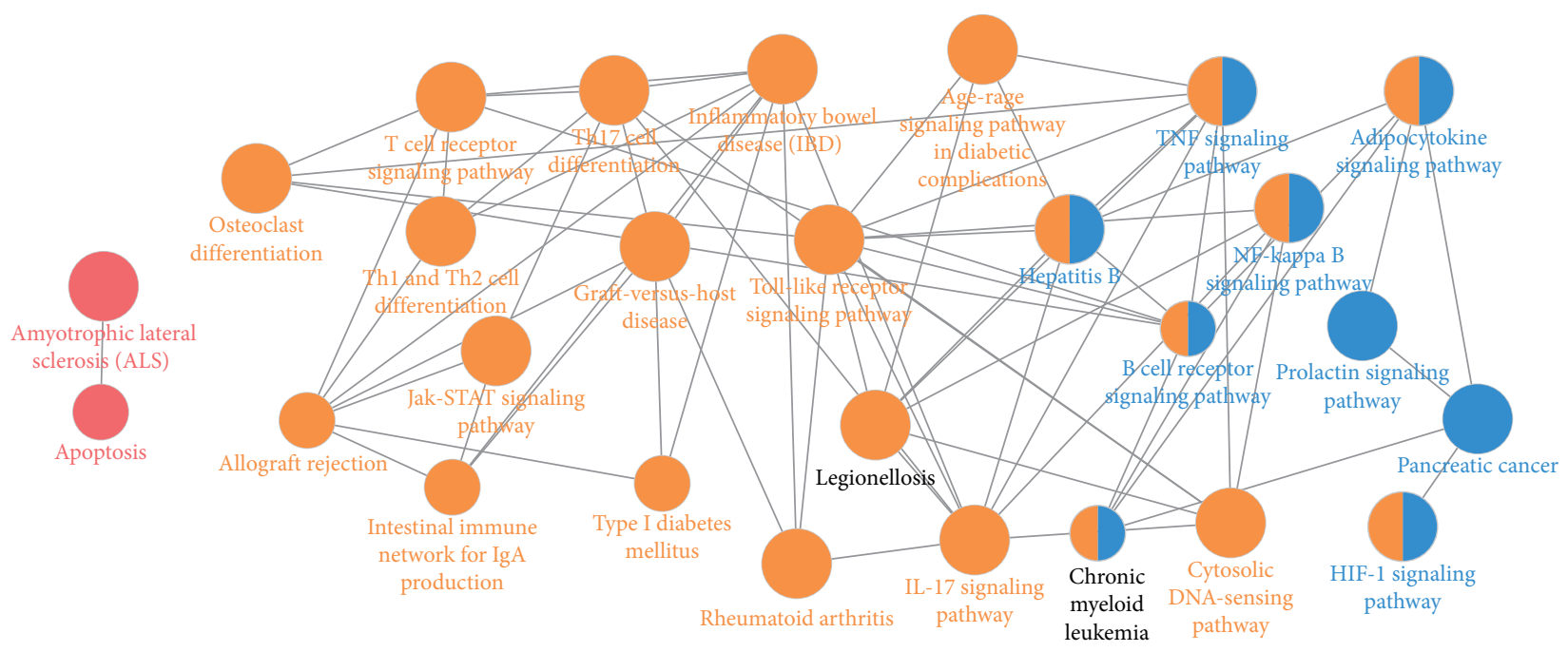

(b)

FIGURE 4: Enrichment analysis of candidate targets for KBL against vitiligo. The enrichment analysis is generated by ClueGo and the most vital term in the group is labeled. Functionally related groups partially overlap. Representative enriched biological process or pathway $(P<0.05)$ interactions among main KBL targets. (a) Candidate KBL targets enriched in the representative biological process are shown. (b) Candidate KBL targets enriched in the representative signaling pathway are shown.

neuronal excitability, and neurosecretion but also inducing the direct death of melanocytes. To sum up, the present outcomes demonstrate the vital pharmacological mechanism of KBL against vitiligo and also shed novel light on the drug exploitation strategy of vitiligo.

\section{Discussion}

Vitiligo, the most prevalent depigmentation skin disease, is caused by dysfunction or destruction of melanocytes, the major pigment-producing cells [52]. Vitiligo is psychologically destructive in general, though it is not physically harmful [53]. The present accessible therapeutic regimens, such as steroid therapy, vitamin D analogs, and excimer laser, are not proper for the majority of patients due to the complicated, ineffective, and time-consuming effects
$[54,55]$. Large-scale GWAS, mainly performed in Europeanderived white and Chinese populations, have reported about 56 different genetic loci contributed to vitiligo risk, part of which also made contribution to oxidative stress, melanogenesis of melanocytes, neuroendocrine modulation, and autoimmunity $[56,57]$. Thus, better understandings of the possible mechanisms might contribute to more specificity and effectiveness of the therapeutic regimens.

The 30-year application KBL has proved its practical effectiveness on vitiligo, however, without systematical understanding of the pharmacological mechanisms. In our study, network pharmacology was employed to further investigate the mechanisms of KBL on vitiligo. To this end, we mainly focused on three aspects. Firstly, common targets of KBL of vitiligo were enriched in pathological changes of intracellular redox equilibrium and ion homeostasis. Secondly, enrichment 
analysis showed the regulatory effects of KBL on the differentiation of T-lymphocytes and that the cytokines produced may be the most important mechanism of vitiligo treatment. Thirdly, neuroendocrine regulation was also considered as the critical sections of KBL on vitiligo therapy. To sum up, the therapeutic effect of $\mathrm{KBL}$ on vitiligo was mediated by controlling the levels of its targets, which have been shown to be critically involved in vitiligo progression in a multicomponent, multitarget, and multilink pattern.

As is known to all, lymphocytes, especially T-lymphocytes along with $\mathrm{T}$ cells-generated cytokines, play crucial roles in vitiligo progression, as indicated by multiple research studies. Due to the advanced inflammation speculations, the imbalance of Th1/Th2 has become a paradigm in vitiligo pathogenesis, which can affect on a variety of inflammatory cytokines, such as ILs $[58,59]$. The latest studies have shown that various immune and inflammatory cells can synthesize and secrete neurotrophic factors via the enhancement of Th2 cells, induction of mast cell proliferation and differentiation, activation of eosinophils, intensification of immune response, and promotion of the secretion of inflammatory mediators. Similarly, we have also demonstrated that there are diverse critical signaling pathways related to T-lymphocytes differentiation (TLRs) [60] regulated by KBL on vitiligo therapy. Furthermore, studies have shown that nervous pathways affect the release of neuropeptides (NPs) and various cell behaviors, subsequently leading to changes on costimulatory molecules and cytokine expression of innate and adaptive immunity in the skin, thereby affecting the pathogenesis and progression of vitiligo $[51,61]$. Collectively, we cautiously speculate that vitiligo might be characterized by systemic neuro-immuno-inflammatory response, which is consistent with the holistic understanding of both physiological and pathological states of the human body in TCM concept.

Despite the valuable discoveries, there are still certain limitations. To begin with, some compounds of herbs in KBL were neglected in consideration of the inadequate data obtained from existing databases and laboratory findings. Secondly, there was a lack of animal assay or clinical trial for validation of our speculations. Thirdly, there is great difficulty in the direct evaluation of specific targets and critical active ingredients, which are retrieved from a chemically complicated as well as cognitively rare TCM formula. Therefore, future studies are warranted to confirm these potential mechanisms of KBL on vitiligo from animal assays and clinical trials. In addition, we aim to intensively explore (1) the upstream and downstream mechanisms of this formula in regulating these pathways and (2) the difference in the material basis in regulating these different pathways.

\section{Data Availability}

The data used to support the findings of this study are available from the corresponding author upon request.

\section{Conflicts of Interest}

The authors declare no conflicts of interest.

\section{Acknowledgments}

This work was supported by the Natural Science Foundation of China (no. 8180150888). The authors thank the members of their laboratory and their collaborators for their research work.

\section{Supplementary Materials}

Table S1: all the candidate compounds of KBL. Table S2: all the potential targets of KBL. Table S3: known vitiligo-related targets. Table S4: KBL shared 15 potential targets with known vitiligo-related targets. Table S5: topological feature values of all/significant/candidate targets for KBL against vitiligo Table S6: detailed information on six existing protein-protein interaction databases. Table S7: gene ontology analysis of the potential targets of KBL. Table S8: GO enrichment analysis of the 15 overlapped (between KBL potential and known vitiligo-related) targets. Table S9: KEGG enrichment analysis of the 15 overlapped (between KBL potential and known vitiligo-related) targets. (Supplementary Materials)

\section{References}

[1] K. Ezzedine, V. Eleftheriadou, M. Whitton, and N. van Geel, "Vitiligo," Lancet, vol. 386, no. 9988, pp. 74-84, 2015.

[2] M. Whitton, M. Pinart, J. M. Batchelor et al., "Evidence-based management of vitiligo: summary of a cochrane systematic review," British Journal of Dermatology, vol. 174, no. 5, pp. 962-969, 2016.

[3] C. Krüger and K. U. Schallreuter, "A review of the worldwide prevalence of vitiligo in children/adolescents and adults," International Journal of Dermatology, vol. 51, no. 10, pp. 1206-1212, 2012.

[4] Y. Wang, S. Li, and C. Li, "Perspectives of new advances in the pathogenesis of vitiligo: from oxidative stress to autoimmunity," Medical Science Monitor, vol. 25, pp. 1017-1023, 2019.

[5] M. I. K. El-Sayed, A. A. A. El-Ghany, and R. R. Mohamed, "Neural and endocrinal pathobiochemistry of vitiligo: comparative study for a hypothesized mechanism," Frontiers in Endocrinology, vol. 9, p. 197, 2018.

[6] N. B. Goldstein, M. I. Koster, L. G. Hoaglin et al., "Narrow band ultraviolet B treatment for human vitiligo is associated with proliferation, migration, and differentiation of melanocyte precursors," Journal of Investigative Dermatology, vol. 135, no. 8, pp. 2068-2076, 2015.

[7] C.-C. E. Lan, G. -S. Chen, M.-H. Chiou, C.-S. Wu, C.-H. Chang, and H.-S. Yu, "FK506 promotes melanocyte and melanoblast growth and creates a favourable milieu for cell migration via keratinocytes: possible mechanisms of how tacrolimus ointment induces repigmentation in patients with vitiligo," British Journal of Dermatology, vol. 153, no. 3, pp. 498-505, 2005.

[8] S. A. Birlea, N. B. Goldstein, and D. A. Norris, "Repigmentation through melanocyte regeneration in vitiligo," Dermatologic Clinics, vol. 35, no. 2, pp. 205-218, 2017.

[9] N. Bagherani, B. R. Smoller, and T. Lotti, "Vitamin D supplementation for vitiligo," Dermatologic Therapy, vol. 30, no. 6, Article ID e12548, 2017.

[10] Y. Fa, Y. Lin, X. J. Chi et al., "Treatment of vitiligo with 308$\mathrm{nm}$ excimer laser: our experience from a 2-year follow-up of 
979 Chinese patients," Journal of the European Academy of Dermatology and Venereology, vol. 31, no. 2, pp. 337-340, 2017.

[11] Y. Konishi, K. Yamanaka, and H. Mizutani, "Treatment of vitiligo vulgaris with the combination therapy of topical steroid and vitamin D3 compound," Dermatology Reports, vol. 4, no. 1, p. e8, 2012.

[12] P. E. Grimes, "Vitiligo. An overview of therapeutic approaches," Dermatologic Clinics, vol. 11, no. 2, pp. 325-338, 1993.

[13] M. Picardo, M. L. Dell'Anna, K. Ezzedine et al., "Vitiligo," Nature Reviews Disease Primers, vol. 1, no. 1, p. 15011, 2015.

[14] S. Li, T.-P. Fan, W. Jia, A. Lu, and W. Zhang, "Network pharmacology in traditional Chinese medicine," EvidenceBased Complementary and Alternative Medicine, vol. 2014, Article ID 138460, 2 pages, 2014.

[15] S. Gianfaldoni, U. Wollina, M. Tirant et al., "Herbal compounds for the treatment of vitiligo: a review," Open Access Macedonian Journal of Medical Sciences, vol. 6, no. 1, pp. 203-207, 2018.

[16] C. Zhang, L. Zhou, J. Huang, and W. Shi, “A combination of Yiqiqubai granule and 308-nm excimer laser in treatment of segmental vitiligo: a prospective study of 233 patients," Journal of Dermatological Treatment, vol. 28, no. 7, pp. 668671, 2017.

[17] T. Pei, C. Zheng, C. Huang et al., "Systematic understanding the mechanisms of vitiligo pathogenesis and its treatment by Qubaibabuqi formula," Journal of Ethnopharmacology, vol. 190, pp. 272-287, 2016.

[18] S. Tian, Y. Li, D. Li et al., "Modeling compound-target interaction network of traditional Chinese medicines for type II diabetes mellitus: insight for polypharmacology and drug design," Journal of Chemical Information and Modeling, vol. 53, no. 7, pp. 1787-1803, 2013.

[19] S. Zhang and Z. Min, "The effect of Kang-Bai-ling for treatment of localized vitiligo on 31 patients," Nanjing University of Chinese Medicine, vol. 3, p. 184, 1997, in Chinese.

[20] M. Xu, Y. Kong, and Z. Min, "The regulative effect of KangBai-ling on TYR and MITF in melanognesis," China Journal of Leprosy and Skin Diseases, vol. 32, no. 6, pp. 335-338, 2016, in Chinese.

[21] R. Zhang, X. Zhu, H. Bai, and K. Ning, "Network pharmacology databases for traditional Chinese medicine: review and assessment," Frontiers in Pharmacology, vol. 10, 2019.

[22] Y. Xiong, Y. Yang, W. Xiong, Y. Yao, H. Wu, and M. Zhang, "Network pharmacology-based research on the active component and mechanism of the antihepatoma effect of Rubia cordifolia L.," Journal of Cellular Biochemistry, vol. 120, no. 8, pp. 12461-12472, 2019.

[23] J. Ru, P. Li, J. Wang et al., "TCMSP: a database of systems pharmacology for drug discovery from herbal medicines," Journal of Cheminformatics, vol. 6, no. 1, p. 13, 2014.

[24] X. Xu, W. Zhang, C. Huang et al., "A novel chemometric method for the prediction of human oral bioavailability," International Journal of Molecular Sciences, vol. 13, no. 6, pp. 6964-6982, 2012.

[25] S. Tian, J. Wang, Y. Li, X. Xu, and T. Hou, "Drug-likeness analysis of traditional Chinese medicines: prediction of druglikeness using machine learning approaches," Molecular Pharmaceutics, vol. 9, no. 10, pp. 2875-2886, 2012.

[26] S. Wang, H. Wang, and Y. Lu, "Tianfoshen oral liquid: a CFDA approved clinical traditional Chinese medicine, normalizes major cellular pathways disordered during colorectal carcinogenesis," Oncotarget, vol. 8, no. 9, pp. 14549-14569, 2017.
[27] G. Kavli, K. Midelfart, J. Raa, and G. Volden, "Phototoxicity from furocoumarins (psoralens) of Heracleum laciniatum in a patient with vitiligo. Action spectrum studies on bergapten, pimpinellin, angelicin and sphondin," Contact Dermatitis, vol. 9, no. 5, pp. 364-366, 1983.

[28] P. Li, J. Chen, J. Wang et al., "Systems pharmacology strategies for drug discovery and combination with applications to cardiovascular diseases," Journal of Ethnopharmacology, vol. 151, no. 1, pp. 93-107, 2014.

[29] N. Rappaport, M. Twik, I. Plaschkes et al., "MalaCards: an amalgamated human disease compendium with diverse clinical and genetic annotation and structured search," Nucleic Acids Research, vol. 45, no. D1, pp. D877-D887, 2017.

[30] A. Hamosh, A. F. Scott, J. Amberger, D. Valle, and V. A. McKusick, "Online Mendelian inheritance in man (OMIM)," Human Mutation, vol. 15, no. 1, pp. 57-61, 2000.

[31] Y. H. Li, C. Y. Yu, X. X. Li et al., "Therapeutic target database update 2018: enriched resource for facilitating bench-to-clinic research of targeted therapeutics," Nucleic Acids Research, vol. 46, no. D1, pp. D1121-D1127, 2018.

[32] D. S. Wishart, Y. D. Feunang, A. C. Guo et al., "DrugBank 5.0: a major update to the DrugBank database for 2018," Nucleic Acids Research, vol. 46, no. D1, pp. D1074-D1082, 2018.

[33] K. G. Becker, K. C. Barnes, T. J. Bright, and S. A. Wang, "The genetic association database," Nature Genetics, vol. 36, no. 5, pp. 431-432, 2005.

[34] A. Martin, M. E. Ochagavia, L. C. Rabasa, J. Miranda, J. Fernandez-de-Cossio, and R. Bringas, "BisoGenet: a new tool for gene network building, visualization and analysis," BMC Bioinformatics, vol. 11, no. 1, 2010.

[35] Y. Tang, M. Li, J. Wang, Y. Pan, and F.-X. Wu, "CytoNCA: a cytoscape plugin for centrality analysis and evaluation of protein interaction networks," Biosystems, vol. 127, pp. 67-72, 2015.

[36] M. Su, C. Guo, M. Liu, X. Liang, and B. Yang, "Therapeutic targets of vitamin $\mathrm{C}$ on liver injury and associated biological mechanisms: a study of network pharmacology," International Immunopharmacology, vol. 66, pp. 383-387, 2019.

[37] G. Bindea, B. Mlecnik, H. Hackl et al., "ClueGO: a Cytoscape plug-in to decipher functionally grouped gene ontology and pathway annotation networks," Bioinformatics, vol. 25, no. 8, pp. 1091-1093, 2009.

[38] L. J. den Hartigh, "Conjugated linoleic acid effects on cancer, obesity, and atherosclerosis: a review of pre-clinical and human trials with current perspectives," Nutrients, vol. 11, no. 2, 2019.

[39] S. Andres, S. Pevny, R. Ziegenhagen et al., "Safety aspects of the use of quercetin as a dietary supplement," Molecular Nutrition \& Food Research, vol. 62, no. 1, 2018.

[40] A. M. Pironi, P. R. de Araujo, M. A. Fernandes, H. R. N. Salgado, and M. Chorilli, "Characteristics, biological properties and analytical methods of ursolic acid: a review," Critical Reviews in Analytical Chemistry, vol. 48, no. 1, pp. 86-93, 2018.

[41] D.-Z. Wei, X.-Y. Guo, L.-N. Lin et al., "Effects of angelicin on ovalbumin (OVA)-Induced airway inflammation in a mouse model of asthma," Inflammation, vol. 39, no. 6, pp. 18761882, 2016.

[42] A. O. Antwi, D. D. Obiri, N. Osafo, L. B. Essel, A. D. Forkuo, and C. Atobiga, "Stigmasterol alleviates cutaneous allergic responses in rodents," BioMed Research International, vol. 2018, Article ID 3984068, 13 pages, 2018. 
[43] X. Zhang, J. Feng, K. Mu et al., "Effects of single herbal drugs on adhesion and migration of melanocytes," Journal of Traditional Chinese Medicine, vol. 25, no. 3, pp. 219-221, 2015.

[44] K. Boniface, A. Taïeb, and J. Seneschal, "New insights into immune mechanisms of vitiligo," Giornale Italiano di Dermatologia e Venereologia, vol. 151, no. 1, pp. 44-54, 2016.

[45] J. E. Harris, "Cellular stress and innate inflammation in organspecific autoimmunity: lessons learned from vitiligo," Immunological Reviews, vol. 269, no. 1, pp. 11-25, 2016.

[46] A. Reichert Faria, J. E. Jung, C. C. Silva de Castro, and L. de Noronha, "Reduced immunohistochemical expression of adhesion molecules in vitiligo skin biopsies," Pathology-Research and Practice, vol. 213, no. 3, pp. 199-204, 2017.

[47] A. Sahoo, B. Lee, K. Boniface et al., "MicroRNA-211 regulates oxidative phosphorylation and energy metabolism in human vitiligo," Journal of Investigative Dermatology, vol. 137, no. 9, pp. 1965-1974, 2017.

[48] H. Xie, F. Zhou, L. Liu et al., "Vitiligo: how do oxidative stressinduced autoantigens trigger autoimmunity?," Journal of Dermatological Science, vol. 81, no. 1, pp. 3-9, 2016.

[49] Y.-M. Jeong, Y.-G. Choi, D.-S. Kim et al., "Cytoprotective effect of green tea extract and quercetin against hydrogen peroxide-induced oxidative stress," Archives of Pharmacal Research, vol. 28, no. 11, pp. 1251-1256, 2005.

[50] R. M. Heriniaina, J. Dong, P. K. Kalavagunta, H. L. Wu, D.-S. Yan, and J. Shang, "Effects of six compounds with different chemical structures on melanogenesis," Chinese Journal of Natural Medicines, vol. 16, no. 10, pp. 766-773, 2018.

[51] T. Lotti, M. Zanardelli, and A. M. D'Erme, "Vitiligo: what's new in the psycho-neuro-endocrine-immune connection and related treatments," Wiener Medizinische Wochenschrift, vol. 164, no. 13-14, pp. 278-285, 2014.

[52] E. H. Kemp, N. G. Gavalas, D. J. Gawkrodger, and A. P. Weetman, "Autoantibody responses to melanocytes in the depigmenting skin disease vitiligo," Autoimmunity Reviews, vol. 6, no. 3, pp. 138-142, 2007.

[53] P. E. Grimes and M. M. Miller, "Vitiligo: patient stories, selfesteem, and the psychological burden of disease," International Journal of Women's Dermatology, vol. 4, no. 1, pp. 32-37, 2018.

[54] A. K. Rzepecki, B. N. McLellan, and N. Elbuluk, "Beyond traditional treatment: the importance of psychosocial therapy in vitiligo," Journal of Drugs in Dermatology, vol. 17, no. 6 , pp. 688-691, 2018.

[55] J. J. Nordlund, "The medical treatment of vitiligo: an historical review," Dermatologic Clinics, vol. 35, no. 2, pp. 107-116, 2017.

[56] Y. Jin, S. A. Birlea, P. R. Fain et al., "Genome-wide association analyses identify 13 new susceptibility loci for generalized vitiligo," Nature Genetics, vol. 44, no. 6, pp. 676-680, 2012.

[57] Y. Jin, G. Andersen, D. Yorgov et al., "Genome-wide association studies of autoimmune vitiligo identify 23 new risk loci and highlight key pathways and regulatory variants," Nature Genetics, vol. 48, no. 11, pp. 1418-1424, 2016.

[58] M. G. Santaguida, S. C. Del Duca, C. Virili, L. Gargano, and M. Centanni, "The presence of non-segmental vitiligo modifies intracellular cytokine subsets in patients with chronic lymphocytic thyroiditis," International Journal of Immunopathology and Pharmacology, vol. 23, no. 4, pp. 1203-1209, 2010.

[59] A. Nouri-Koupaee, P. Mansouri, H. Jahanbini, M. H. Sanati, and Z. Jadali, "Differential expression of mRNA for T-bet and GATA-3 transcription factors in peripheral blood mononuclear cells of patients with vitiligo," Clinical and Experimental Dermatology, vol. 40, no. 7, pp. 735-740, 2015.

[60] T. Traks, M. Keermann, M. Karelson et al., "Polymorphisms in toll-like receptor genes are associated with vitiligo," Frontiers in Genetics, vol. 6, 2015.

[61] A. Miniati, Z. Weng, B. Zhang, A. J. Stratigos, E. Nicolaidou, and T. C. Theoharides, "Neuro-immuno-endocrine processes in vitiligo pathogenesis," International Journal of Immunopathology and Pharmacology, vol. 25, no. 1, pp. 1-7, 2012. 


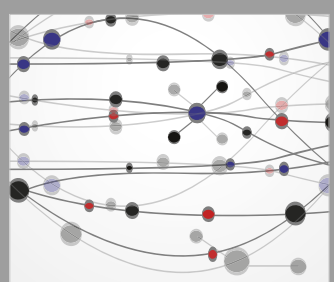

The Scientific World Journal
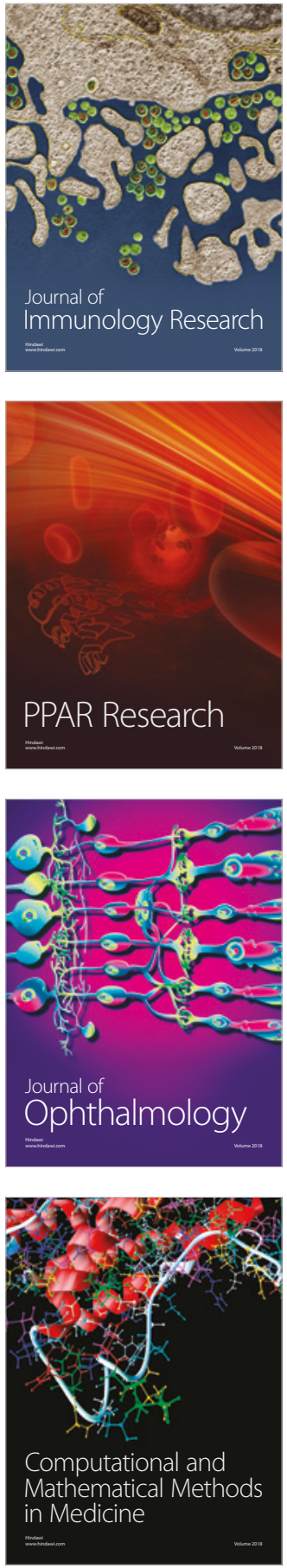

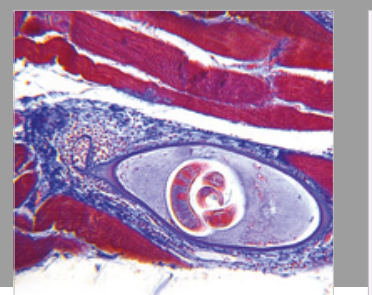

Gastroenterology Research and Practice

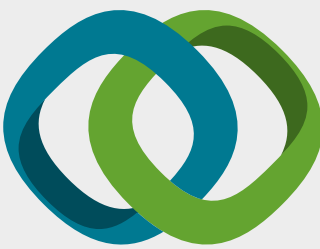

\section{Hindawi}

Submit your manuscripts at

www.hindawi.com
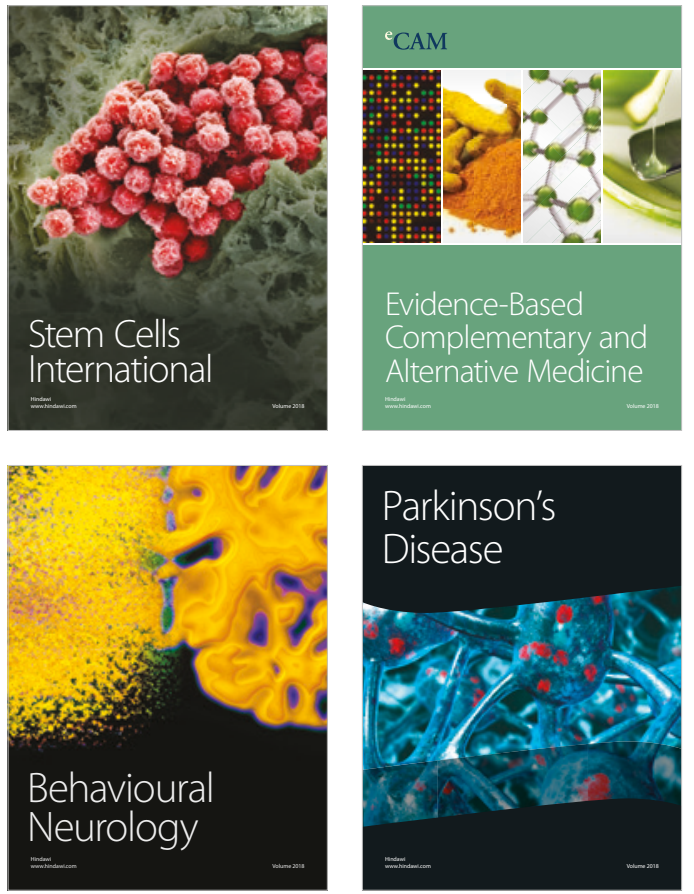

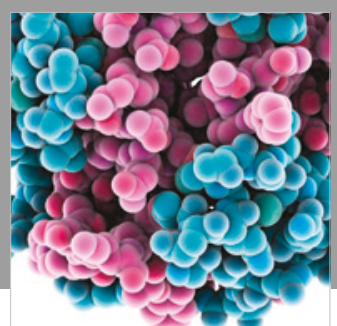

ournal of

Diabetes Research

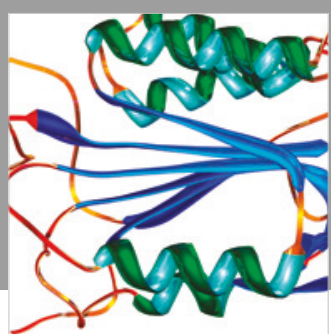

Disease Markers
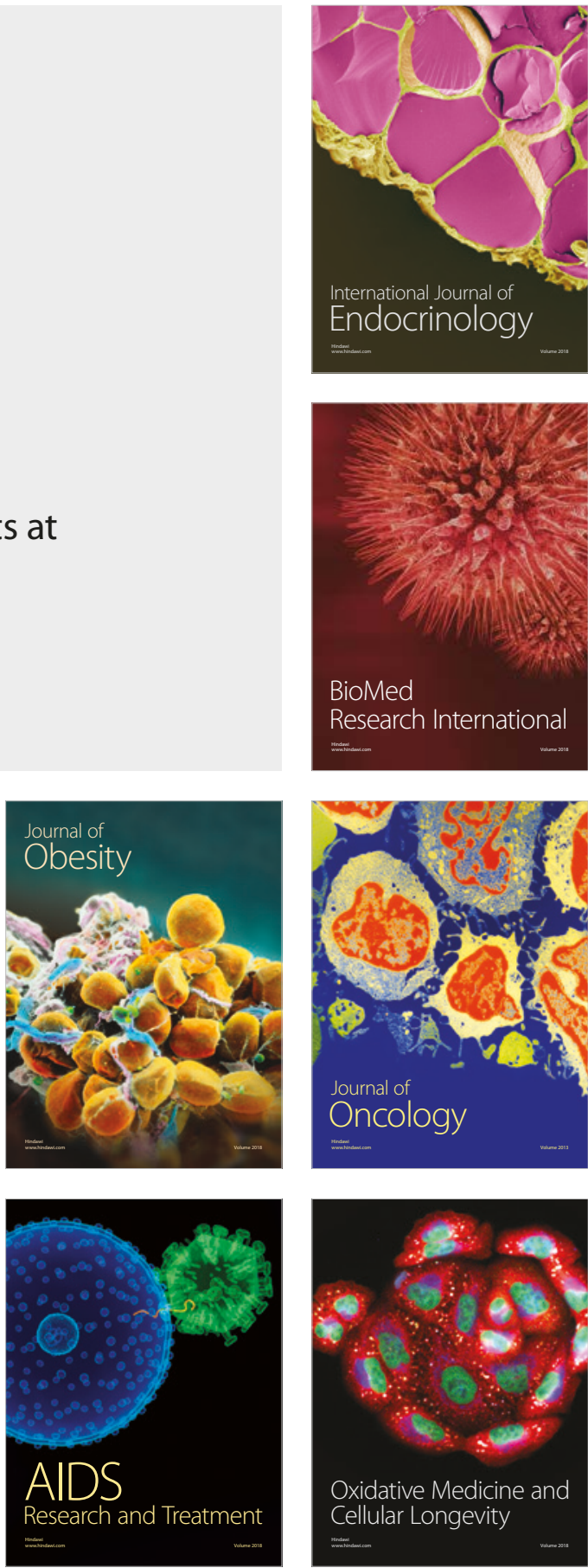